\title{
Data Sequencing for Minimum-transition Transmission
}

\author{
Rajeev Murgai and Masahiro Fujita \\ Fujitsu Laboratories of America, Inc. \\ Santa Clara, California, USA, \{murgai, fujita\}@fla.fujitsu.com
}

\author{
Sriram C. Krishnan \\ Department of EECS, University of California at Berkeley \\ Berkeley, California,USA, krishnan@eecs.berkeley.edu
}

\begin{abstract}
Given a set of data words or messages to be transmitted over a bus such that the sequence (order) in which they are transmitted is irrelevant, we address the problem of determining the optimum sequence that minimizes the total number of transitions on the bus. Since busses take up significant fraction of chip-area, the bus capacitances are often considerable; then the bus power may account for as much as $40 \%$ of the total power consumed on the chip (Designer 1995). Thus exploiting the freedom to resequence the data words can lead to substantial power savings.

This problem arises during the flushing of a cache and transmission of packets over a channel. We also show how some power minimization problems in scheduling during highlevel synthesis, in instruction-sequencing for embedded applications, and in die testing can be cast as the data ordering problem. We prove that the data ordering problem is NPcomplete. Nevertheless, we propose two polynomial-time algorithms to approximate the optimum solution to within a constant factor. The first algorithm gives a solution to within a factor of 2 from the optimum, and the second within a factor of 1.5 , but at an additional cost that is a function of the word-size. Experimental results confirm that resequencing data using the proposed algorithms leads to significant reduction (by $36 \%$ ) in switching activity and hence power savings.
\end{abstract}

Keywords: Low Power Design, System-Level Design.

\section{MOTIVATION}

With the proliferation of portable electronic devices, low power has become an important design objective. Among the suite of emerging techniques for low power designs are pipelining and parallelization (Chandrakasan, Sheng \& Brodersen 1992, Duncan, Swamy \& Jain 1993), reduction of supply voltage (Chandrakasan, Sheng \& Brodersen 1992), and minimization of switching activity. The problem of minimizing the switching activity in a circuit has been addressed at various levels: system level (Chandrakasan, Sheng \& Brodersen 1992, Bunda, Athas \& Fussell 1994, Chandrakasan, Potkonjak, Rabaey \& Brodersen 1992), logic or gate level (Roy \& Prasad 1992, Shen, Devadas, White, Ghosh \& Keutzer 1993, Tsui, Pedram \& Despain 1993, Tiwari, Ashar \& Malik 1993), layout level (Chao \& Wong 1994, Hirendu \& Pedram 1993, Cong, Koh \& Leung 1994), transistor level (Tan \& Allen 1994), etc. In this paper, we examine the problem of reducing switching activity on high-capacitance bus 
lines. In several applications such as microprocessors and DSPs, busses account for almost $40 \%$ of the chip area; $30-40 \%$ of the power consumption on a chip is due to bus-power (Designer 1995), i.e., the power consumed in charging/discharging the often large bus capacitances.

More precisely, given a set of data words or messages to be transmitted over a bus such that the sequence in which they are transmitted is irrelevant, we address the problem of determining the optimum sequence that minimizes the total number of transitions on the bus. Consider the following situations where resequencing (or reordering) can lead to power reduction.

Cache write-back: Consider a computer system with a main memory and a cache. On a context switch, often the cache (main memory) is "flushed," i.e. the data residing in the cache (main memory) has to be written back to main memory (secondary memory). Or, in a distributed memory multi-processor system the entire contents of a local cache may be written back over a global bus to memory and the local caches of other processors. In all such applications the order in which the lines are written back is often irrelevant. For minimizing the power dissipated during the transmission, the problem is: in which order should the words be transferred such that the bus lines have the fewest transitions ( 0 to 1 or 1 to 0 )? Not only should one consider transitions on the data bus, but also on the address bus. Since average power consumed is directly proportional to the expected switching activity, fewer transitions result in lower power consumption.

Example 1 Consider 3 cache locations, with the following addresses and data.

\begin{tabular}{cc}
\hline address & data \\
\hline$a_{0}=0111$ & $w_{0}=0001$ \\
$a_{1}=1000$ & $w_{1}=1110$ \\
$a_{2}=1001$ & $w_{2}=0011$ \\
\hline
\end{tabular}

If the order in which the data is transferred from the cache is $w_{0}, w_{1}, w_{2}$, we can compute the total number of transitions on the address and the data lines as follows. For $w_{0}$ to $w_{1}$, there are 4 transitions on the address bus, and 4 transitions on the data bus. Thus, in all 8 transitions. Similarly, from $w_{1}$ to $w_{2}$, there is 1 transition on the address bus and 3 on the data bus, making it a total of 4. For the entire transmission, the number of transitions is then 12. However, if the data is transferred in the order $w_{0}, w_{2}, w_{1}$, the number of transitions reduces to $4+4=8$, two-thirds of the previous count.

The problem then is to determine the order in which the data words should be transferred such that the total number of transitions on the data and the address busses are minimized.

Scheduling in high-level synthesis: Consider the scheduling problem in high-level synthesis, where high-level operations have to be scheduled in different clock cycles subject to resource constraints. For instance, consider an FIR (finite-impulse response) filter that computes $x_{0} c_{0}+x_{1} c_{1}+x_{2} c_{2}+x_{3} c_{3}$, where $x_{i}$ s are signal inputs, and $c_{i} s$ are fixed constants. Assume we are constrained to use only one multiplier for all the four multiplications. We will need to use multiplexors/busses at the two inputs of the multiplier that will select appropriate $x_{i}$ s and $c_{i}$ s corresponding to the multiplication operations in different clock cycles. Since in the FIR computation, multiplications can be executed in any order, there is an opportunity to schedule them on the single available multiplier so that the total number of transitions at the multiplier inputs is minimized over the four multiplications. ${ }^{*}$ Consider the multiplier input fed by the constants $c_{i} s$. If the multiplications are scheduled as $x_{0} c_{0}$ followed by $x_{1} c_{1}$, followed by $x_{2} c_{2}$, and then $x_{3} c_{3}$, the transitions seen at this multiplier input over one sample period will be the sum of the number of bits switching from $c_{0}$ to $c_{1}$, $c_{1}$ to $c_{2}, c_{2}$ to $c_{3}$, and $c_{3}$ to $c_{0}$. The $c_{3}$ to $c_{0}$ transition is considered because the next sample

* It has been observed that the transitions within the multiplier are positively correlated to the transitions at the multiplier inputs. 


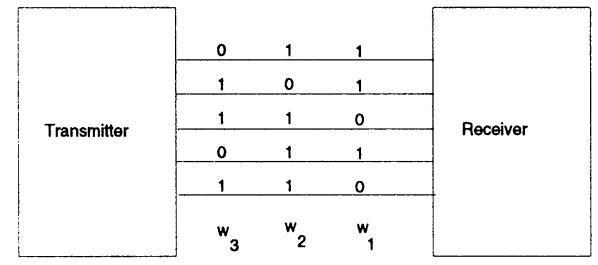

Figure 1 Transmitting a set of words

period starts with the multiplication $x_{0} c_{0}$. For a different schedule, the transitions will be different. Thus we discover the problem of word sequencing (the words are the constants $\left.c_{i} s\right)$ in this context. Note that for the second input of the multiplier, which is fed by the input signals $x_{i}$, it is not possible to determine the transitions a priori, since the inputs can assume any values. It may be possible to run several simulation inputs and compute the average number of transitions between $x_{i}$ and $x_{j}$. Then, the scheduling can be carried out so as to minimize the number of transitions at both inputs.

Instruction sequencing: In compiled code, there often arise sets of instructions that can be executed in any order without altering the behavior of the program. Therefore, for low power applications, it is possible to resequence the instructions, thereby reducing power consumption in fetching the instructions from memory (Tiwari, Malik \& Wolfe 1994).

Die testing: The first steps of IC manufacturing involve etching the dies on a wafer and testing them. The defective dies are removed and the good ones are packaged. During testing, input vectors are applied to the bare die, which has very little capacity for power dissipation. Thus the die is stressed much more during this step than at any other step in its life-time (Chakravarty \& Dabholkar 1994), and may fail during test session, thus reducing the die-yield. This reduction is significant, especially in larger and higher density circuits such as multi-chip modules. This leads to the problem of power minimization during die testing. One way to lower power consumption is to reorder the test vectors (for combinational circuits) and test sequences (for sequential circuits) so that the switching activity in the die is minimized. Since switching activities at the circuit inputs and within the circuit are strongly correlated, it is worthwhile to minimize the activity at the circuit inputs, say by reordering the tests. A modified version of the test ordering problem was addressed in (Chakravarty \& Dabholkar 1994), where the power consumed in the circuit under test is also explicitly modeled.

Transmitting a set of records: Consider a transmitter-receiver system, transmitting and receiving a set of data records over a bus (see Figure 1). There are applications where it does not matter in what order the records are sent. For instance, if each record carries its own identification, the ordering of the records can be arbitrary (the receiver figures out which record is which by examining the identification label of the record). However, to minimize power dissipation during transmission, it may be that one ordering is better than another. It is useful that the records be sent in an order that minimizes the total number of transitions over the entire set of records. ${ }^{*}$

Underlying all the above scenarios is the problem of determining the optimum order in which to transmit data words such that the total number of transitions is minimized. Optimization for low power by exploiting the freedom to order the items to be transmitted is a hitherto unstudied and unutilized optimization possibility; the savings resulting can be substantial - especially for transmission over busses, since bus capacitances, especially off-chip, can be very large.

We distinguish two kinds of situations. In certain applications such as instruction sequencing and transmitting a set of records, the data to be transmitted is known and fixed $a$ priori, unlike in some other applications, such as cache write-back, where the data changes at different time instances. The algorithms we propose can be used in both cases, but they would be more effective in the first case, where the algorithms can be simply applied to

* However, in many applications, each record is a set of data words and not a single word. This issue is addressed in Section 7. 


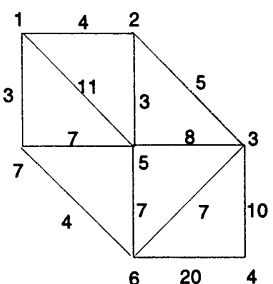

(A)

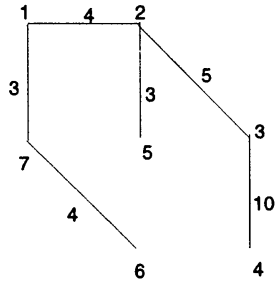

(B)

Figure 2 An example graph and its minimum-weight spanning tree

reorder the data statically, without incurring any area and power cost (this is akin to optimization at compile-time). However, when the data is not known a priori, the algorithms have to be implemented in silicon and then invoked dynamically, akin to optimization at run-time. The extra area and power consumption of this silicon can be justified only if the bus capacitance and the frequency of transmission are high enough-as in the case of data-intensive, distributed-memory applications.

The rest of the paper is organized as follows. In Section 2 we formally state the data ordering problem for minimum transitions. Graph-theoretic preliminaries are covered in Section 3. Section 4 addresses the complexity of the data ordering problem for minimum total transitions. We prove that the corresponding decision problem is NP-complete. In Section 5, we present three heuristics to solve the problem. Experimental results and implementation details are covered in Section 6. Section 7 extends the basic data ordering formulation to some other applications. Proofs not provided in this paper can be found in (Murgai, Fujita \& Krishnan 1995).

\section{PROBLEM DEFINITION}

We are given a set of data words $w_{1}, w_{2}, \ldots, w_{n}$, each $k$-bit long, that are to be transmitted over a bus. The goal is to minimize the total number of transitions over the entire transmission interval.

If word $w_{r}$ is transmitted, immediately followed by $w_{s}$, the total number of transitions is given by the number of bits that change. This is $d\left(w_{r}, w_{s}\right)=\sum_{j=1}^{k} w_{r j} \oplus w_{s j}$ sometimes called the Hamming distance between $w_{r}$ and $w_{s}$. Here, $w_{r j}$ denotes the $j^{\text {th }}$ bit of $w_{r}$. Here $\oplus$ denotes the EX-OR operation. For instance, Hamming distance between 11001 and 10010 is 3 , i.e., $d(11001,10010)=3$.

This data ordering problem (DOP) can be restated as: "find a permutation $\sigma$ of the words $\left\{w_{1}, w_{2}, \ldots, w_{n}\right\}$ such that the total number of transitions $\sum_{i=1}^{n-1} d\left(w_{\sigma(i)}, w_{\sigma(i+1)}\right)$ is minimized."

\section{PRELIMINARIES}

We give a few definitions and basic results from graph theory that will be used in the paper. The reader who is familiar with them may skip this section.

A spanining tree of a graph $G=(V, E)$ is a subgraph of $G$ that is a tree and spans all the vertices of $G$. If the edges have weights, it makes sense to talk of the minimum-weight spanning tree of $G$, where the weight of a tree is the sum of the weights of the edges in the tree. It turns out that a simple, greedy algorithm of starting from an empty tree and 


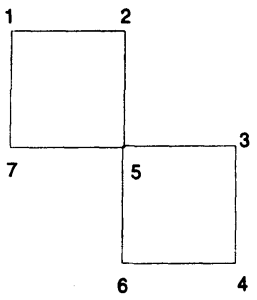

(A)

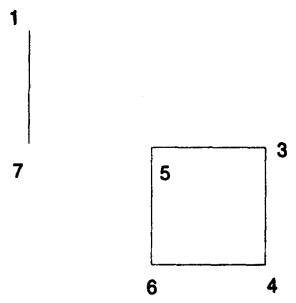

(B)

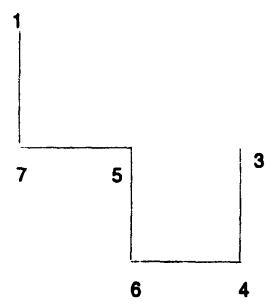

(C)

\section{Figure 3 Determining an Eulerean cycle}

repeatedly selecting the minimum-weight edge and adding it to the partially generated tree if the tree still remains acyclic generates a minimum-weight spanning tree (Nemhauser \& Wolsey 1988). For the weighted graph of Figure 2 (A), the minimum-weight spanning tree generated by this algorithm is shown in Figure $2(\mathrm{~B})$.

A Hamiltonian tour of a graph $G=(V, E)$ is a walk (simple path) with the same beginning and end points that visits each vertex of $V$ exactly once. A Hamiltonian path of $G$ is a walk with different beginning and end points that visits each vertex of $V$ exactly once. For instance, 1, 2, 3, 4, 6, 5, 7, 1 is a Hamiltonian tour of the graph of Figure 2 (A), whereas $1,2,3,4,6,5,7$ is a Hamiltonian path. If the edges have weights, we have the problems of finding a minimum-weight Hamiltonian tour (better known as the traveling salesman problem tour or TSP-tour) and minimum-weight Hamiltonian path (TSP-path). Both of these are known to be NP-complete (Garey \& Johnson 1979), even for complete graphs.

A graph $G=(V, E)$ in which there may be more than one edge joining a pair of nodes is called a multigraph. An Eulerean cycle of a multigraph is a walk with the same beginning and end points that contains each edge of the graph exactly once. For example, in Figure 3 , the tour $1,2,5,3,4,6,7,1$ is an Eulerean cycle. The following classical result gives necessary and sufficient conditions for the existence of an Eulerean cycle. $A$ multigraph contains an Eulerean cycle if and only if each node is of even degree. The following simple procedure finds an Eulerean cycle in such a multigraph.

Procedure 1 (Finding an Eulerean cycle) Start at any vertex $v$ of $G$. Traverse any edge incident on $v$ whose removal does not disconnect the remaining (untraversed) graph. ${ }^{*}$ Let this edge be $(v, w)$. Delete $(v, w)$. Repeat the procedure from $w$. Terminate when no more edges remain in the graph. This can happen only when at $v$. The order in which the edges are traversed yields the Eulerean cycle.

Consider the graph $G$ of Figure 3 (A). Since all the vertices are of even degrees, $G$ has an Eulerean cycle. Let us arbitrarily start from vertex 1 . Since removal of edge $(1,2)$ leaves $G$ connected, we come to vertex 2 , and delete the edge $(1,2)$. Similarly, from 2 , we go to vertex 5 and delete $(2,5)$. Now, we have a choice of whether to traverse the edge $(5,7)$ or $(5,3)$. If we traverse $(5,7)$, the remaining graph is disconnected, as shown in Figure 3 (B). However, if we traverse edge $(5,3)$, the remaining graph is still connected, as shown in Figure $3(C)$. So, we traverse $(5,3)$. Thereafter, there are no more choices to exercise. The Eulerean cycle is then $1,2,5,3,4,6,5,7,1$.

Given a complete graph $G=(V, E)$ and a spanning tree $T=\left(V, E^{\prime}\right)$ of $G$, the following procedure constructs a Hamiltonian tour on $G$.

Procedure 2 (Constructing a Hamiltonian tour) Construct the multigraph $\tilde{G}$ from $T$ by duplicating each edge e $\in E^{\prime}$. Since each node of $\widetilde{G}$ is of even degree, $\widetilde{G}$ contains an Eulerean

*If initially each node in $G$ is of even degree, the algorithm always finds such an edge. 
cycle $U$. Construct $U$ using Procedure 1. Delete all the node repetitions from $U$ except for the final return to the first node. The resulting node sequence HT is a Hamiltonian tour on $G$.

Note that any permutation of vertices of a complete graph yields a Hamiltonian tour. However, we will be interested in a minimum-weight Hamiltonian tour, for which we will start from a minimum-weight spanning tree $T$ and use Procedure 2.

Let $d(S)$ denote the total weight of the tour (path, tree) $S$, where the weight of a tour (path, tree) $S$ is the sum of the weights of the edges in $S$.

If there is a non-negative weight $d\left(v_{i}, v_{j}\right)$ on the edge $\left(v_{i}, v_{j}\right)$ of $G$, and if in addition, the weights satisfy triangle inequality, then it is easy to see that $d(H T) \leq d(U)$. This is because while obtaining $H T$ from $U$, all the node repetitions are being deleted. For instance, if $v_{1} v_{3} v_{4} v_{1} v_{6}$ is a subsequence of $U$, deleting the second occurrence of $v_{1}$ is equivalent to replacing the edges $\left(v_{4}, v_{1}\right)$ and $\left(v_{1}, v_{6}\right)$ by $\left(v_{4}, v_{6}\right)$. Because the triangle inequality is satisfied, $d\left(v_{4}, v_{1}\right)+d\left(v_{1}, v_{6}\right) \geq d\left(v_{4}, v_{6}\right)$. Hence, the weight of the tour $H T$ is no more than the weight of the tour $U$. Also, $d(U)=2 d(T)$. So we get,

$d(H T) \leq 2 d(T)$

We will use this fact later.

A matching in a graph $G=(V, E)$ is a subset $E^{\prime} \subseteq E$ such that no two edges of $E^{\prime}$ are incident to each other. A perfect matching is a matching that is incident to each vertex in $V$. Clearly, $|V|$ must be even for a perfect matching to exist. In many cases, the edges of the graph have weights on them. One optimization problem is to find a minimum-weight (perfect) matching in $G$. There exists an algorithm for this problem that runs in time $O\left(n^{3}\right)$ (Papadimitriou \& Steiglitz 1982).

Consider the graph of Figure 2 (A). It does not have a perfect matching, since it has odd number of vertices. However, $\{(1,2),(3,5),(4,6)\}$ is a matching, whereas $\{(1,2),(3,5),(3,4)$, $(6,7)\}$ is not, since edges $(3,5)$ and $(3,4)$ share the vertex 3 .

\section{DATA ORDERING PROBLEM IS NP-COMPLETE}

In this section, we show that the data ordering problem for minimum number of transitions is hard.

As a warm-up, we first show that DOP can be transformed to the minimum-weight TSP-path (or Hamiltonian path) problem in a complete graph $G$ (which is known to be NP-complete (Garey \& Johnson 1979)). Construct a complete graph $G=(V, E)$, whose vertices $v_{i}$ are in one-to-one correspondence with the data words $w_{i}$. Edge $\left(v_{i}, v_{j}\right)$ has weight $d\left(w_{i}, w_{j}\right)$, the Hamming distance between the words $w_{i}$ and $w_{j}$. Then, the minimum-weight TSP-path in $G$ yields a permutation of the vertices and thus of the data words, for fewest transitions.

This, however, does not imply that DOP is difficult. The edge weights in the general TSP-path problem can be arbitrary, whereas they are not arbitrary in the case of DOP since they are derived from the codes of the vertices, i.e., the data words (see Section 5).

The Data Ordering Problem (DOP) stated as a decision problem is:

INSTANCE: A set of $n k$-bit data words, $w_{1}, w_{2}, \ldots, w_{n}$, where each $w_{i} \in\{0,1\}^{k}$ where $k$ is a positive integer, and a positive integer $L$.

QUESTION: Is there a permutation $\sigma$ of $w_{1}, w_{2}, \ldots, w_{n}$, i.e. a $1-1$ function $\sigma:\{1, \ldots, n\} \rightarrow$ $\{1, \ldots, n\}$ such that $\sum_{i=1}^{n-1} d\left(w_{\sigma(i)}, w_{\sigma(i+1)}\right) \leq L$ ?

Proposition 1 DOP is NP-complete.

Proof. DOP is easily seen to be in NP. 
We transform a special case of the MANHATTAN PATH-TSP problem (Papadimitriou 1977) to DOP. MANHATTAN PATH-TSP can be stated as follows:

INSTANCE: A set $P \subseteq Z^{+} \times Z^{+}$of points in the plane, a positive integer $B .^{*}$

QUESTION: Is there a TSP-path of length $B$ or less in the associated complete graph on $|P|$ vertices, where the distance between the vertices corresponding to the points $\left(x_{1}, y_{1}\right)$ and $\left(x_{2}, y_{2}\right)$ is $\left|x_{1}-x_{2}\right|+\left|y_{1}-y_{2}\right|$ ?

Note that the Manhattan distance between two points corresponds to the sum of the horizontal and the vertical distances between them.

The special case we consider is called MANHATTAN PATH-TSP POLYNOMIAL COORDINATES. It is as follows:

INSTANCE: A set $P \subseteq Z^{+} \times Z^{+}$of $p$ points in the plane, a positive integer $B$. The $x$ and $y$ coordinates of each point have values at most $c p$, where $c$ is a constant.

QUESTION: Is there a TSP-path of length $B$ or less in the associated complete graph on $p$ vertices, where the distance between the vertices corresponding to the points $\left(x_{1}, y_{1}\right)$ and $\left(x_{2}, y_{2}\right)$ is $\left|x_{1}-x_{2}\right|+\left|y_{1}-y_{2}\right|$ ?

MANHATTAN PATH-TSP POLYNOMIAL COORDINATES is NP-complete. It follows from the NP-completeness proof of MANHATTAN PATH-TSP proposed by Papadimitriou (Papadimitriou 1977). The instance of MANHATTAN PATH-TSP he creates in the proof is actually an instance of MANHATTAN PATH-TSP POLYNOMIAL COORDINATES: it satisfies the polynomial-coordinate property.

From MANHATTAN PATH-TSP POLYNOMIAL COORDINATES instance, we will generate a DOP instance consisting of $|P|$ data words, one for each point in $P$. Each data word will have $\max _{i}\left\{x_{i}\right\}+\max _{j}\left\{y_{j}\right\}=X+Y$ bits, and will essentially be a concatenation of the unary codes of the $x$ and the $y$ coordinates of the corresponding point. For example, if $x_{1}=6, y_{1}=4$, and $X=\max _{i}\left\{x_{i}\right\}=8, Y=\max _{i}\left\{y_{i}\right\}=5$, the data word $w_{1}$ will be the concatenation of 00111111 (which is the unary representation of 6 - two zeros to the left are added to make all data words of equal length) and 01111 (which is the unary representation of 4 modulo the padded zeros), i.e., 0011111101111 . It can be easily seen that the Manhattan distance between two points in the set $P$ is the same as the Hamming distance between the corresponding data words. Hence, a TSP-path of length $B$ or less exists if and only if there exists a permutation with associated total number of transitions at most $B$ (thus, the DOP parameters are $n=|P|, k=X+Y, L=B$ ). Note that this is a polynomial-time transformation. In particular, note that since for any point $(x, y)$ in $P, x, y \leq c p$, we obtain $X, Y \leq c p$. Thus, the length of each data word is at most $2 c p$, a polynomial in the size of the MANHATTAN PATH-TSP POLYNOMIAL COORDINATES instance. This completes the proof of NP-completeness of DOP.

\section{APPROXIMATION ALGORITHMS}

As shown in the last section, the data ordering problem is intractable. However, we adapt well-known approximation algorithms to provide solutions with guarantees of closeness to the optimum.

The key to guaranteeing the bounds is that the Hamming distance $d$ satisfies the triangle inequality, i.e., if $w_{1}, w_{2}, w_{3}$ are three data words, then $d\left(w_{1}, w_{2}\right)+d\left(w_{2}, w_{3}\right) \geq d\left(w_{1}, w_{3}\right)$. We make use of this in the sequel.

The approximation algorithms we propose are adaptations of the well-known algorithms proposed for finding a minimum-weight TSP tour (or minimum-weight Hamiltonian tour) when the weights satisfy the triangle inequality (Nemhauser \& Wolsey 1988). As pointed out in Section 4, DOP can be formulated in terms of the minimum-weight Hamiltonian path. Thus, we have to suitably transform minimum-weight tour algorithms to minimum-weight path algorithms.

"The original problem is stated with $P \subseteq Z \times Z$, but there is no loss of generality in replacing $Z$ with $Z^{+}$. $Z^{+}$denotes the set $\{0,1,2, \ldots\}$. 


\subsection{Double Spanning Tree (DST) Heuristic}

1. Construct a complete graph $G=(V, E)$, where each vertex $v_{i}$ corresponds to the data word $w_{i}$, and the weight of the edge $\left(v_{i}, v_{j}\right)$ is $d\left(w_{i}, w_{j}\right)$.

2. Find a minimum-weight spanning tree $T$ in $G$.

3. Apply Procedure 2 to find a Hamiltonian tour $H T$. This procedure first duplicates each edge of $T$, finds an Eulerean cycle $U$ using Procedure 1, and then constructs $H T$ from $U$.

4. Delete the longest edge $e$ in $H T$ to get a Hamiltonian path $H P$. The sequence of vertices in this path corresponds to the desired permutation. In fact, there are two permutations depending on the starting point for the sequence, and both work.

Let $d(S)$ denote the total weight of the tour (path, tree) $S$, as defined in Section 3.

Proposition 2 Let $H P^{*}$ be the optimum (i.e., minimum-weight) Hamiltonian path in $G$. Then, $d(H P) \leq \frac{2(n-1)}{n} d\left(H P^{*}\right)$, where $n$ is the number of the vertices in $G$, and $H P$ is the Hamiltonian path produced by the double spanning tree heuristic.

Thus, the permutation generated by the double spanning tree heuristic comes within a factor of $\frac{2(n-1)}{n}$ of the optimum.

\subsection{Spanning Tree/Minimum Matching (ST-MM) Heuristic}

This heuristic also first constructs a minimum-weight spanning tree. However, to construct an Eulerean cycle, instead of duplicating each edge of the tree, it tries to add fewer edges. It works as follows.

1. Construct a complete graph $G=(V, E)$, where the vertices $v_{i}$ correspond to the data words $w_{i}$, and the weight of the edge $\left(v_{i}, v_{j}\right)$ is $d\left(w_{i}, w_{j}\right)$.

2. Find a minimum-weight spanning tree $T$ in $G$.

3. Identify the set $O$ of vertices in the tree $T$ with odd degrees. There must be an even number of such vertices.

4. Find a minimum-weight perfect matching $M$ on $G(O)=(O, E(O))$, the subgraph of $G$ induced on $O$.* Such a matching exists, since $|O|$ is even and $G(O)$ is a complete graph.

5. Consider the graph $T \cup M$. Since each edge of $M$ connects two different odd-degree vertices of $T, T \cup M$ is a connected graph in which all vertices have even degrees. This implies that there exists an Eulerean cycle $U$ in $T \cup M$. Construct $U$ using Procedure 1. From $U$, construct a Hamiltonian tour $H T$ by deleting the node repetitions, exactly as in Procedure 2. Delete the longest edge $e$ in this tour to get a Hamiltonian path $H P$. This path corresponds to the desired permutation.

Proposition 3 Let $H P^{*}$ be the optimum Hamiltonian path in $G$. Then, $d(H P) \leq \frac{n-1}{n}\left[\frac{3}{2} d\left(H P^{*}\right)+\frac{k}{2}\right]$, where $k$ is the number of bits in a data word, and HP is the Hamiltonian path produced by the spanning tree/perfect matching heuristic.

\subsection{Greedy Heuristic}

The $D S T$ and the $S T$-MM heuristics first form a minimum-weight spanning tree, then an Eulerean cycle, and finally a Hamiltonian path. In contrast, the greedy heuristic is very

* $G(O)$ is a subgraph of $G$ on the vertices $O$ and has exactly those edges of $G$ that have both their end-points in $O$. 
simple. It always works with a path $P$ and at every step extends it towards a Hamiltonian path.

It works as follows. Like the previous heuristics, it also constructs the complete graph $G$. Then it selects the minimum-weight edge, say $(i, j)$, of $G$. The path $P$ after this step is $(i, j)$. Next, the minimum-weight edge incident on an end-point of $P$ (i.e., on $i$ or $j$ ) is selected. Let it be $(i, k)$. Thus $P$ becomes $(k, i, j)$. Thus, at each step the heuristic greedily selects the minimum-weight edge incident on an end-point of $P$ that is not incident on an internal vertex of $P$. The heuristic terminates when all the vertices of $G$ are in $P$, in which case a Hamiltonian path has been constructed. As the experimental results in Section 6 will show, the greedy technique performs the best.

We note that other algorithms used for TSP can also be applied. Some of these are nearest neighbor, nearest insertion, $m$-exchange, etc. (Nemhauser \& Wolsey 1988). However, no performance bounds are known with these heuristics.

\section{EXPERIMENTAL RESULTS}

The objective of our experiments is to evaluate the effectiveness of the proposed resequencing algorithms in minimizing the switching activity. The experimental set-up is as follows. For a set of $n k$-bit data words chosen from a uniform distribution, we count the total number of transitions using the following resequencing schemes:

- random: Words are transmitted in the same order as they were generated. Since the words were generated randomly, we call this scheme random.

- greedy: Words are transmitted in an order determined by the greedy heuristic of Section 5.3.

- DST: Words are transmitted in an order determined by the double spanning tree heuristic of Section 5.1.

- $S T-M M$ : Words are transmitted in an order determined by the spanning tree-minimum matching heuristic of Section 5.2.

For each $n$, we consider different values of $k$.

The results are shown in Table 1. Each entry in the columns corresponding to the four schemes is the total number of transitions for transmitting the entire set of $n$ words. The greedy scheme performs the best. On average, it yields $36 \%$ fewer transitions than random. Average power being directly proportional to the expected number of transitions, $36 \%$ fewer transitions translates to a power saving of $36 \%$ on the busses. Since bus-power can be as high as $40 \%$ of the power consumed on the chip, we can expect about $15 \%$ reduction in the total power.

On average, DST and $S T-M M$ are respectively $29 \%$ and $33 \%$ better than random. Although theoretically $D S T$ and $S T$-MM have a predictable worst-case performance, they are not as good as greedy. The reason is as follows. Both DST and ST-MM construct an Eulerean cycle $U$ before constructing the Hamiltonian path. The final result is sensitive to the choice of $U$. In our implementation, we pick one amongst several possible Eulerean cycles of the graph. In the future, we plan to characterize the sensitivity of the final Hamiltonian path with respect to the starting Eulerean cycle.

One nice implication of the simple greedy scheme being the best is that for applications where the data words are not known a priori (such as in the cache write-back application and inputs $x_{i}$ of the multiplier in the scheduling application), the hardware will not be very complex. Also, the power overhead will be small, since the capacitance driven by this extra hardware is negligible as compared to the bus capacitance.

\section{EXTENSIONS AND CONCLUSIONS}

We consider some applications where the previous heuristics can be applied, with minor modifications.

In the cache write-back problem of Section 1, the transitions on both the address and 


\begin{tabular}{rrrrrr}
\hline$n=$ \# words & $k$ = word-length & random & greedy & DST & ST-MM \\
\hline 10 & 5 & 22 & 10 & 11 & 10 \\
10 & 10 & 46 & 23 & 29 & 23 \\
10 & 20 & 87 & 72 & 74 & 78 \\
10 & 40 & 190 & 149 & 157 & 157 \\
\hline 40 & 5 & 99 & 21 & 26 & 25 \\
40 & 10 & 192 & 87 & 106 & 94 \\
40 & 20 & 390 & 225 & 248 & 243 \\
40 & 40 & 784 & 567 & 608 & 591 \\
40 & 60 & 1171 & 893 & 955 & 923 \\
\hline 80 & 10 & 371 & 149 & 191 & 162 \\
80 & 20 & 780 & 426 & 484 & 455 \\
80 & 40 & 1555 & 1075 & 1192 & 1131 \\
80 & 80 & 3135 & 2439 & 2620 & 2502 \\
80 & 100 & 3919 & 3163 & 3351 & 3233 \\
\hline 100 & 10 & 496 & 174 & 220 & 190 \\
100 & 20 & 1006 & 515 & 618 & 558 \\
100 & 40 & 2001 & 1326 & 1450 & 1373 \\
100 & 80 & 4019 & 3049 & 3335 & 3185 \\
100 & 100 & 4969 & 3894 & 4162 & 3981 \\
100 & 150 & 7482 & 6139 & 6437 & 6348 \\
\hline 200 & 20 & 1998 & 957 & 1221 & 1051 \\
200 & 40 & 4077 & 2490 & 2878 & 2646 \\
200 & 80 & 7916 & 5767 & 6291 & 6053 \\
200 & 100 & 9906 & 7520 & 8289 & 7847 \\
200 & 150 & 15001 & 12027 & 12955 & 12407 \\
200 & 200 & 19996 & 16463 & 17477 & 16881 \\
\hline total & & 91608 & 69620 & 75385 & 72147 \\
\hline & & & & &
\end{tabular}

Table 1 Effect of Data Resequencing on Number of transitions \# words number of randomly generated data words word-length length of each data word random greedy $D S T$ $S T-M M$ total total \# of transitions using a random ordering total \# of transitions using a greedy ordering total \# of transitions using ordering by DST heuristic total \# of transitions using ordering by ST-MM heuristic sum of \# of transitions over all the examples 
the data busses have to be collectively minimized. This is simply done by concatenating the address and the corresponding data and treating them together as a super word, and applying the previous algorithms on the super words.

Next consider the problem of scheduling for high-level synthesis, as described in Section 1. For the FIR filter example, note that the problem of scheduling the multiplications is not that of finding a minimum-weight TSP-path, but rather that of finding a minimum-weight TSP-tour. This is due to the cyclic nature of scheduling: after all the multiplications have been performed in a sample period, they are performed in the same order in the next sample period. Thus we have to include the number of transitions between the $c_{i}$ corresponding to the last multiplication in the current sample period and the $c_{j}$ corresponding to the first multiplication in the next sample period (which is the same as the first multiplication in the current sample period). For the TSP-tour of minimum weight, the approximation algorithms described in (Nemhauser \& Wolsey 1988) can be used directly. These are the same as those in Section 5, except that the final result is the tour HT.

Some other simple extensions are transmitting a set of records where each record is a set of bytes (instead of being a single byte), the bus having a fixed initial and/or final state, etc.

We are currently studying the utility of resequencing in situations where the data is not known a priori, for instance, during cache write-back. One way to solve the problem is to invoke the algorithm when the system is actually running - implemented in hardware. However, it is hard to estimate in advance the associated area, timing, and power penalties. On the other hand, in some applications, such as the CDFG data binding/scheduling problem (Dasgupta \& Karri 1995), it may be possible to estimate the switching activity by using the input signal probabilities. In such cases, the problem becomes identical to that in which the data is known.

We have left unaddressed applications where the data cannot be re-ordered: it has to be transmitted in the same order it is given. Here we may be able to re-encode the data at the transmitter and suitably decode it at the receiver to minimize the transitions over the channel. We are currently working on this problem.

\section{REFERENCES}

Bunda, J., Athas, W. C. \& Fussell, D. (1994). Evaluating Power Implications of CMOS Microprocessor Design Decisions, International Workshop on Low Power Design, pp. 147-152.

Chakravarty, S. \& Dabholkar, V. (1994). Minimizing Power Dissipation in Scan Circuits During Test Application, International Workshop on Low Power Design, pp. 51-56.

Chandrakasan, A., Potkonjak, M., Rabaey, J. \& Brodersen, R. W. (1992). HYPER-LP: A System for Power Minimization Using Architectural Transformations, Proceedings of the International Conference on Computer-Aided Design, pp. 300-303.

Chandrakasan, A., Sheng, T. \& Brodersen, R. W. (1992). Low Power CMOS Digital Design, Journal of Solid State Circuits, pp. 473-484.

Chao, K. Y. \& Wong, D. F. (1994). Low Power Considerations in Floorplan Design, International Workshop on Low Power Design, pp. 45-50.

Cong, J., Koh, C. K. \& Leung, K. S. (1994). Wiresizing with Driver Sizing for Performance and Power Optimization, International Workshop on Low Power Design, pp. 81-86.

Dasgupta, A. \& Karri, R. (1995). Simultaneous Scheduling and Binding for Power Minimization During Microarchitecture Synthesis, International Symposium on Low Power Design, pp. 69-74.

Designer, E. M. (1995). Personal communication.

Duncan, P., Swamy, S. \& Jain, R. (1993). Low-Power DSP Circuit Design Using Retimed Maximally Parallel Architectures, Proceedings of the $1^{\text {st }}$ Symposium on Integrated Systems, pp. 266-275.

Garey, M. R. \& Johnson, D. S. (1979). Computers and Intractability: A Guide to the Theory of NP-completeness, W. H. Freeman and Company.

Hirendu, V. \& Pedram, M. (1993). PCUBE: A Performance Driven Placement Algorithm for Lower Power Designs, Proceedings of Euro-DAC, pp. 72-77. 
Murgai, R., Fujita, M. \& Krishnan, S. C. (1995). Data Sequencing for Minimum-transition Transmission, Internal Report, Fujitsu Labs of America, Inc.

Nemhauser, G. L. \& Wolsey, L. A. (1988). Integer and Combinatorial Optimization, WileyInterscience.

Papadimitriou, C. H. (1977). The Euclidean Traveling Salesman Problem is NP-complete, Theoretical Computer Science 4: 237-244.

Papadimitriou, C. H. \& Steiglitz, K. (1982). Combinatorial Optimization: Algorithms and Complexity, Prentice-Hall, Englewood Cliffs, N. J.

Roy, K. \& Prasad, S. (1992). SYCLOP: Synthesis of CMOS Logic for Low Power Applications, Proceedings of the Int'l Conference on Computer Design: VLSI in Computers and Procesors, pp. 464-467.

Shen, A., Devadas, S., White, J., Ghosh, A. \& Keutzer, K. (1993). A Combinational Logic Design Methodology Targeting Low Power Applications, MIT Technical Report (available from the authors).

Tan, C. H. \& Allen, J. (1994). Minimization of Power in VLSI Circuits Using Transistor Sizing, Input Ordering, and Statistical Power Estimation, International Workshop on Low Power Design, pp. 75-80.

Tiwari, V., Ashar, P. \& Malik, S. (1993). Technology Mapping for Low Power, Proceedings of the $30^{\text {th }}$ Design Automation Conference, pp. 74-79.

Tiwari, V., Malik, S. \& Wolfe, A. (1994). Power Analysis of Embedded Software: A First Step towards Software Power Minimization, Proceedings of the International Conference on Computer-Aided Design, pp. 384-390.

Tsui, C.-Y., Pedram, M. \& Despain, A. M. (1993). Technology Decomposition and Mapping Targeting Low Power Dissipation, Proceedings of the $30^{\text {th }}$ Design Automation Conference, pp. 68-73.

\section{BIOGRAPHY}

Rajeev Murgai received B.Tech. in Electrical Engineering from Indian Institute of Technology, New Delhi, India in 1987, M.S. in Electrical and Computer Engineering from Carnegie Mellon University, Pittsburgh, PA in 1989, and Ph.D. in Electrical Engineering and Computer Sciences from the University of California at Berkeley in 1993. In 1994, he joined Fujitsu Laboratories of America, Inc., California, where he is currently a Member of Research Staff. His research interests include FPGAs, logic synthesis, circuit partitioning, low power design, logic simulation, and formal verification.

He was awarded the President of India Gold Medal for standing first in the class of 1987 at the Indian Institute of Technology, New Delhi, India. He received best paper nomination in the Design Automation Conference in 1992. He has authored the book Logic Synthesis for Field-programmable Gate Arrays.

Masahiro Fujita received all of his academic degrees from the University of Tokyo. He received B.S. in Electrical Engineering in 1980, M.S. in Information Engineering in 1982, and Ph.D. in Information Engineering in 1985. His Ph.D. thesis advisor was Prof. Tohru Moto-oka, chairman of the Japanese Fifth Generation Computer Project. From 1985 to 1993 he was employed as a research scientist by Fujitsu Laboratories in Kawasaki, Japan. Currently, he is director of Advanced CAD at Fujitsu Laboratories of America in Santa Clara, CA. He received the Sakai Award from the Information Processing Society of Japan in 1994. He has been on program committees for many conferences dealing with digital design. He is an Associate Editor of the IEEE Transactions on Computer-Aided Design and Formal Methods on Systems Design. His primary interest is in Computer-Aided Design of digital systems, especially logic synthesis and verification.

Sriram C. Krishnan is a Ph.D. student in Electrical Engineering and Computer Science at the University of California at Berkeley. He is currently completing his dissertation titled " $\omega$-automata, games, and synthesis," under the supervision of Prof. Robert K. Brayton. His research interests include computer-aided design and verification of Integrated Circuits, as well as algorithms and game theory. 